\title{
Successful Chemotherapy with Carboplatin and S-1 for Thymic Carcinoma: A Case Report
}

\author{
Satoshi Igawa ${ }^{a}$ Nobuyuki Yanagisawa $^{b}$ Mikiko Ishihara $^{a}$ \\ Michiko Kimura $^{a}$ Sachiyo Maki ${ }^{a}$ Sakiko Otani ${ }^{a}$ Jiichiro Sasaki ${ }^{a}$ \\ Noriyuki Masuda ${ }^{a}$ \\ Departments of ${ }^{\mathrm{a}}$ Respiratory Medicine and ${ }^{\mathrm{b}}$ Pathology, Kitasato University School of \\ Medicine, Sagamihara, Japan
}

\section{Key Words}

Thymic carcinoma $\cdot$ Carboplatin $\cdot$ S-1

\begin{abstract}
Thymic carcinoma is a rare but aggressive neoplasm. Although there is no clearly optimal first- or second-line chemotherapy regimen for thymic carcinoma, platinum-based chemotherapy has repeatedly been shown to be of benefit to patients with advanced thymic carcinoma. Some case reports have described S-1 as a novel agent with good activity against advanced thymic carcinoma. A 74-year-old female was diagnosed with thymic carcinoma complicated by pleural dissemination and pericardial effusion of carcinomatosa. She was treated with carboplatin on day 1 plus S-1 on days 1-14 in cycles repeated every 3 or 4 weeks. Four cycles of this regimen were administered, and a partial response was confirmed. There were no severe hematological or nonhematological toxicities, and no dose reduction was necessary. To our knowledge, this is the first report to demonstrate the efficacy of combination chemotherapy consisting of carboplatin and S-1 against thymic carcinoma.
\end{abstract}

(C) 2013 S. Karger AG, Basel

\section{Introduction}

Thymic carcinoma is a rare, invasive neoplasm, and its clinical course tends to be more aggressive than that of thymoma [1-3]. The extent of disease at presentation often precludes complete surgical resection, and systemic chemotherapy plays a very important role in 
Igawa et al:: Successful Chemotherapy with Carboplatin and S-1 for Thymic Carcinoma: A Case Report

treatment. Because of its rare occurrence, only small numbers of cases have been analyzed in previous studies published thus far [4-7]. The optimal chemotherapy regimen for thymic carcinoma remains unclear. Previous case reports have described S-1 as a novel agent with good activity against advanced thymic carcinoma [8-10]. Here, we report a case of advanced thymic carcinoma in which there was a partial response to combination chemotherapy consisting of carboplatin plus S-1.

\section{Case Report}

A 74-year-old female was diagnosed with thymic carcinoma in October 2012. Magnetic resonance imaging (MRI) of the chest revealed a large anterior mediastinal mass, pleural dissemination, and pericardial effusion (fig. 1a). Immediate pericardiocentesis improved her cardiopulmonary distress. A CT-guided percutaneous biopsy of the anterior mediastinal mass was done and small tissue fragments were obtained. Histologically, the tumor was composed of epithelioid cells with high cellularity, which had nuclear atypia and eosinophilic cytoplasm in fibrotic stroma (fig. 2a). Lymphoid cell infiltration was not evident in the tumor. Immunohistochemically, tumor cells were positive for epithelial markers such as AE1/3 and CK5/6. In addition, CD5 (fig. 2b) and CD117 (fig. 2c) were also detected in tumor cells. Neuroendocrine markers including chromogranin A and synaptophysin were negative. The tumor cells showed a high MIB-1 labeling index (approximately 30\%). These findings were consistent with thymic carcinoma. According to the Masaoka classification, the patient had unresectable, locally advanced disease (IVa). In consideration of the complication of the pericardial effusion and the fact that the patient had adequate organ function, we initially treated her with carboplatin plus paclitaxel. However, an allergic reaction to paclitaxel occurred. In November 2012, the patient provided written informed consent before treatment and she was started on carboplatin (AUC, 5) plus oral S-1 $\left(40 \mathrm{mg} / \mathrm{m}^{2}\right.$ twice per day) on days 1-14 as second-line chemotherapy in cycles which were repeated every 3 or 4 weeks. After 2 cycles of this regimen, MRI of the chest revealed a 32\% decrease in the diameter of the anterior mediastinal tumor and a reduction of the pericardial effusion, indicating a partial response based on the RECIST criteria (fig. 1b). The partial response was confirmed after 4 cycles of this regimen (fig. 1c). Hematologic toxicity consisted of grade 3 neutropenia and grade 1 thrombocytopenia, and nonhematologic toxicity consisted of grade 2 nausea, grade 2 anorexia, and grade 1 fatigue. No dose reduction was necessary. Since the completion of treatment with carboplatin plus oral S-1, the patient has remained well, and no disease progression has been observed up until the time of writing this case report (September 2013).

\section{Discussion}

Since several reports have indicated that combination chemotherapy regimens such as carboplatin and paclitaxel [4-6], and carboplatin and amurubicin [7] are effective against unresectable thymic carcinoma, a carboplatin-containing regimen is a valid treatment option, the same as ADOC (cisplatin, doxorubicin, vincristine, and cyclophosphamide) and CODE (cisplatin, vincristine, doxorubicin, and etoposide). This report describes successful chemotherapy with carboplatin plus S-1 in a patient with unresectable thymic carcinoma. S1 is an oral fluoropyrimidine agent that consists of tegafur, 5-chloro-2, 4-dihydroxypyridine (gimeracil; CDHP), and potassium oxonate (Oxo) in a molar ratio of 1:0.4:1 [11, 12]. CDHP 


\begin{tabular}{l|l}
\hline DOI: $10.1159 / 000356143$ & $\begin{array}{l}\text { C 2013 S. Karger AG, Basel } \\
\text { www.karger.com/cro }\end{array}$ \\
\hline
\end{tabular}

Igawa et al.: Successful Chemotherapy with Carboplatin and S-1 for Thymic Carcinoma: A Case Report

causes the serum 5-fluorouracil (5-FU) concentration to rise by competitively inhibiting dihydropyrimidine dehydrogenase, which catalyzes the degradation of 5-FU, thereby increasing serum 5-FU concentrations. S-1 is expected to act more intensively than older fluoropyrimidine agents, but higher 5-FU concentrations in the intestinal mucosa can lead to severe gastrointestinal toxicities. Oxo prevents diarrhea by selectively inhibiting orotate phosphoribosyltransferase. Therefore, gastrointestinal toxicity during intensive S-1 treatment is reduced by including Oxo. A phase 3 trial demonstrated noninferiority of carboplatin plus S-1 to paclitaxel plus carboplatin as first-line treatment for advanced nonsmall cell lung cancer [13]. Also, updated results of the above study established the efficacy of carboplatin and S-1 for patients with squamous cell carcinoma as well as for patients with non-squamous carcinoma [14]. Several case reports have described S-1 as a novel agent with good activity against advanced thymic carcinoma [8-10].

\section{Conclusion}

It is reasonable to say that an S-1-containing regimen such as carboplatin plus S-1 may be effective against thymic carcinoma. To our knowledge, this is the first report to demonstrate the efficacy of carboplatin plus S-1 chemotherapy against thymic carcinoma. Because of the activity of this chemotherapy regimen against thymic carcinoma and the rare occurrence of this disease, a multi-institutional prospective study to evaluate this treatment option is warranted.

\section{Disclosure Statement}

None of the authors report any conflict of interest.

\section{References}

1 Shimosato Y, Kameya T, Nagai K, Suemasu K: Squamous cell carcinoma of the thymus: an analysis of eight cases. Am J Surg Pathol 1991;1:109-121.

2 Suster S, Rosai J: Thymic carcinoma. A clinicopathologic study of 60 cases. Cancer 1991;67:1025-1032.

-3 Yano M, Sasaki H, Yokoyama T, Yukiue H, Kawano O, Suzuki S, Fujii Y: Thymic carcinoma: 30 cases at a single institution. J Thoracic Oncol 2008;3:265-269.

4 Lemma GL, Lee JW, Aisner SC, Langer CJ, Tester WJ, Johnson DH, Loehrer PJ Sr: Phase II study of carboplatin and paclitaxel in advanced thymoma and thymic carcinoma. J Clin Oncol 2011;29:2060-2065.

5 Igawa S, Murakami H, Takahashi T, Nakamura Y, Tsuya A, Naito T, et al: Efficacy of chemotherapy with carboplatin and paclitaxel for unresectable thymic carcinoma. Lung Cancer 2010;67:194-197.

6 Takeda K, Hirai F, Yamanaka T, Taguchi K, Daga H, Shimizu J, et al: Multicenter prospective study of carboplatin and paclitaxel for advanced thymic carcinoma: West Japan Oncology Group 4207L (abstract 7529). J Clin Oncol 2013;31(suppl).

7 Kawashima Y, Inoue A, Sugawara S, Harada M, Kobayashi K, Kozuki T, et al: Phase II study of amrubicin (AMR) and carboplatin (CBDCA) for invasive thymoma (IT) and thymic carcinoma (TC): NJLCG0803 (abstract 7530). J Clin Oncol 2013;31(suppl).

8 Ono A, Naito T, Yamamoto N: S-1 treatment for chemorefractory thymic carcinoma. J Thorac Oncol 2008;3:1076.

9 Okuma Y, Shimokawa T, Takagi Y, Hosomi Y, Iguchi M, Okamura T, et al: S-1 is an active anticancer agent for advanced thymic carcinoma. Lung Cancer 2010;70:357-363.

10 Koizumi T, Agatsuma T, Komatsu Y, Kubo K: Successful S-1 monotherapy for chemorefractory thymic carcinoma. Anticancer Res 2011;31:299-301.

11 Shirasaka T, Nakano K, Takechi T, Satake H, Uchida J, Fujioka A, et al: Activity of 1 M tegafur-0.4 M 5-chloro2,4-dihydroxypyridine-1 M potassium oxonate (S-1) against human colon carcinoma orthotopically implanted into nude rats. Cancer Res 1996;56:2602-2606. 


\section{Case Reports in Oncology}

\begin{tabular}{l|l}
\hline Case Rep Oncol 2013;6:526-530 \\
\hline DOI: $10.1159 / 000356143$ & $\begin{array}{l}\text { C 2013 S. Karger AG, Basel } \\
\text { www.karger.com/cro }\end{array}$ \\
\hline
\end{tabular}

Igawa et al.: Successful Chemotherapy with Carboplatin and S-1 for Thymic Carcinoma: A Case Report

12 Okamoto I, Fukuoka M: S-1: a new oral fluoropyrimidine in the treatment of patients with advanced nonsmall-cell lung cancer. Clin Lung Cancer 2009;10:290-294.

13 Okamoto I, Yoshioka H, Morita S, Ando M, Takeda K, Seto T, et al: Phase III trial comparing oral S-1 plus carboplatin with paclitaxel plus carboplatin in chemotherapy-naïve patients with advanced non-small-cell lung cancer: results of a west Japan oncology group study. J Clin Oncol 2010;28:5240-5246.

14 Yoshioka H, Okamoto I, Morita S, Ando M, Takeda K, Seto T, et al: Efficacy and safety analysis according to histology for S-1 in combination with carboplatin as first-line chemotherapy in patients with advanced nonsmall-cell lung cancer: updated results of the West Japan Oncology Group LETS study. Ann Oncol 2013;24:1326-1331.
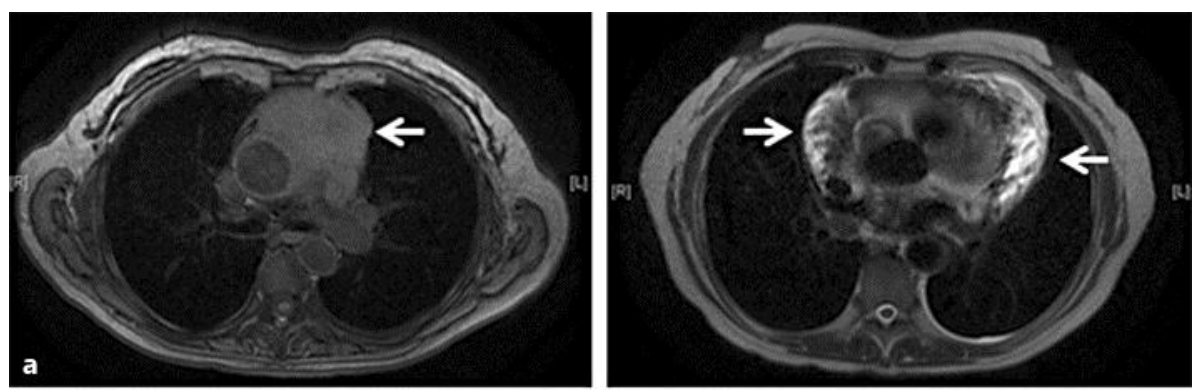

Before chemotherapy

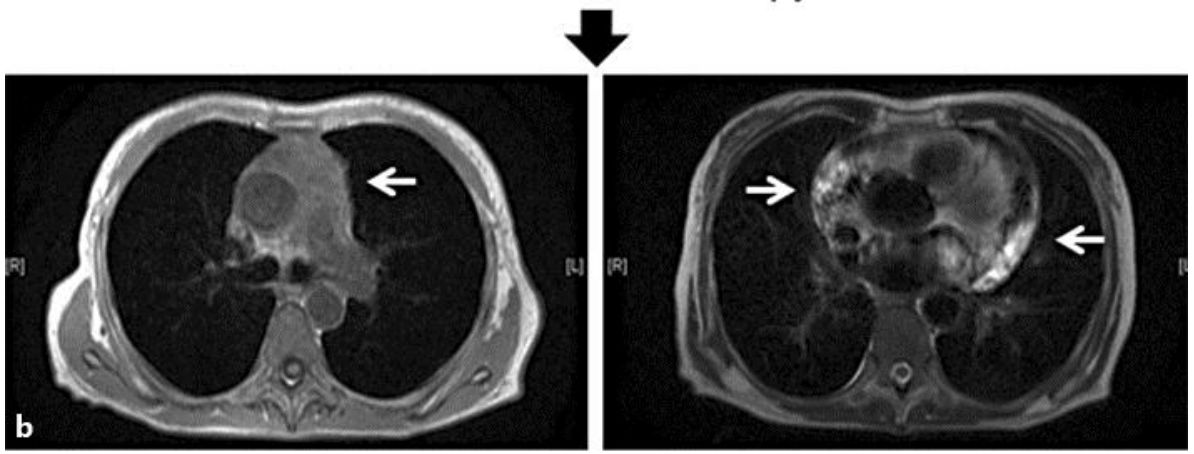

After 2 cycles of chemotherapy

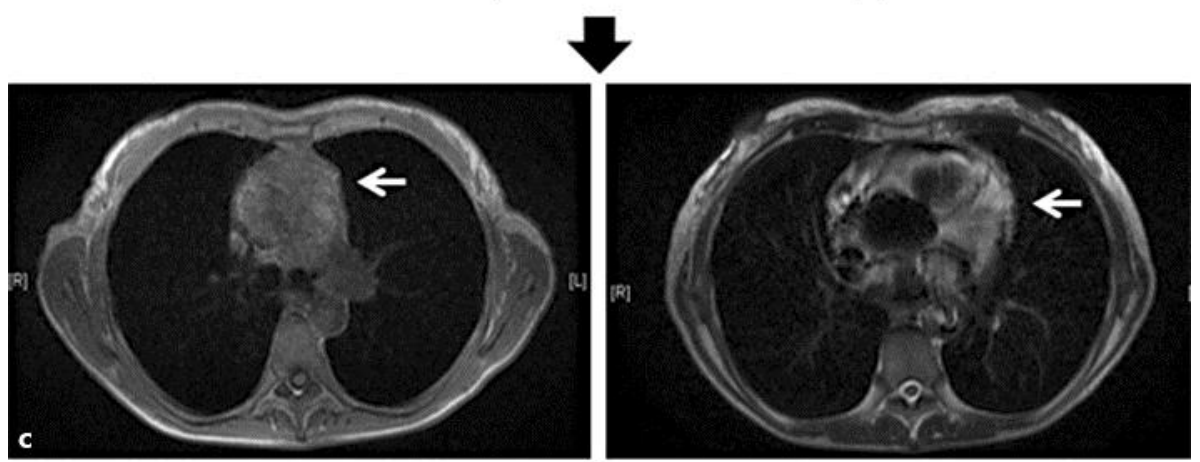

After 4 cycles of chemotherapy

Fig. 1. MRI of the chest before chemotherapy (a), after 2 cycles of chemotherapy (b), and after 4 cycles of chemotherapy (c). An anterior mediastinal mass and pericardial effusion can be seen (arrows; a); the size of the mass and volume of the pericardial effusion were reduced after the chemotherapy (arrows; $\mathbf{b}, \mathbf{c}$ ). 


\section{Case Reports in Oncology}

Igawa et al.: Successful Chemotherapy with Carboplatin and S-1 for Thymic Carcinoma: A Case Report

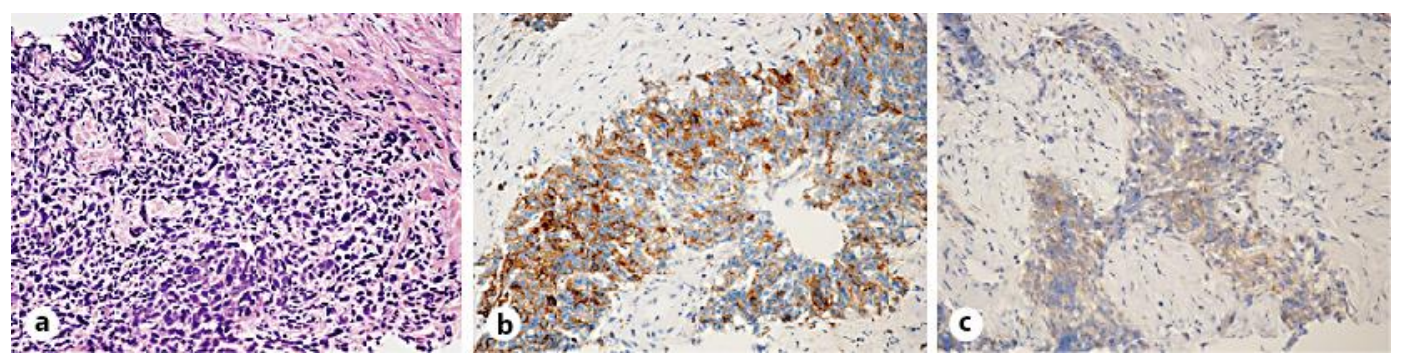

Fig. 2. a Section of the tumor composed of epithelioid cells with high cellularity. The tumor cells were positive for CD5 (b) and CD117 (c). HE. Original magnification, $\times 200$. 